\title{
Digenic mutations account for variable phenotypes in idiopathic hypogonadotropic hypogonadism
}

\author{
Nelly Pitteloud, ${ }^{1}$ Richard Quinton, ${ }^{2,3}$ Simon Pearce, ${ }^{2,4}$ Taneli Raivio, ${ }^{1}$ James Acierno, ${ }^{1}$ \\ Andrew Dwyer, ${ }^{1}$ Lacey Plummer, ${ }^{1}$ Virginia Hughes, ${ }^{1}$ Stephanie Seminara, ${ }^{1}$ Yu-Zhu Cheng, ${ }^{2,4}$ \\ Wei-Ping Li, ${ }^{2,4}$ Gavin Maccoll, ${ }^{5}$ Anna V. Eliseenkova, ${ }^{6}$ Shaun K. Olsen, ${ }^{6}$ Omar A. Ibrahimi, ${ }^{6}$ \\ Frances J. Hayes, ${ }^{1}$ Paul Boepple, ${ }^{1}$ Janet E. Hall, ${ }^{1}$ Pierre Bouloux, ${ }^{5}$ \\ Moosa Mohammadi, ${ }^{6}$ and William Crowley $\mathrm{Jr}^{1}$
}

${ }^{1}$ Reproductive Endocrine Unit of the Department of Medicine and Harvard Reproductive Endocrine Science Centers, Massachusetts General Hospital, Boston, Massachusetts, USA. ${ }^{2}$ Department of Endocrinology and ${ }^{3}$ Royal Victoria Infirmary, School of Clinical Medical Sciences, and ${ }^{4}$ Institute for Human Genetics, University of Newcastle upon Tyne, Newcastle upon Tyne, United Kingdom. ${ }^{5}$ Department of Endocrinology, Royal Free Hospital, London, United Kingdom. ${ }^{6}$ Department of Pharmacology, New York University School of Medicine, New York, New York, USA.

\begin{abstract}
Idiopathic hypogonadotropic hypogonadism (IHH) due to defects of gonadotropin-releasing hormone $(\mathrm{GnRH})$ secretion and/or action is a developmental disorder of sexual maturation. To date, several singlegene defects have been implicated in the pathogenesis of IHH. However, significant inter- and intrafamilial variability and apparent incomplete penetrance in familial cases of IHH are difficult to reconcile with the model of a single-gene defect. We therefore hypothesized that mutations at different IHH loci interact in some families to modify their phenotypes. To address this issue, we studied 2 families, one with Kallmann syndrome (IHH and anosmia) and another with normosmic IHH, in which a single-gene defect had been identified: a heterozygous FGF receptor 1 (FGFR1) mutation in pedigree 1 and a compound heterozygous gonadotropin-releasing hormone receptor (GNRHR) mutation in pedigree 2, both of which varied markedly in expressivity within and across families. Further candidate gene screening revealed a second heterozygous deletion in the nasal embryonic LHRH factor (NELF) gene in pedigree 1 and an additional heterozygous FGFR1 mutation in pedigree 2 that accounted for the considerable phenotypic variability. Therefore, 2 different gene defects can synergize to produce a more severe phenotype in IHH families than either alone. This genetic model could account for some phenotypic heterogeneity seen in GnRH deficiency.
\end{abstract}

\section{Introduction}

Genetic analyses of idiopathic hypogonadotropic hypogonadism ( $\mathrm{IHH})$, an important human disease model with implications for the discovery of genes responsible for human puberty, have provided considerable insight into the genes that control sexual maturation. IHH is a clinically and genetically heterogenous disorder resulting in gonadotropin-releasing hormone $(\mathrm{GnRH})$ deficiency that can be inherited as an X-linked, autosomal recessive, or autosomal dominant trait. IHH has been considered to be a monogenic disorder with several loci identified to date: Kallmann syndrome 1 sequence (KAL1) (1-3), FGF receptor 1 (FGFR1) (4), prokineticin 2 (PROK2), and prokineticin receptor 2 (PROKR2) (5) underlie cases of Kallmann syndrome (KS), while gonadotropin-releasing hormone receptor (GNRHR) (6), FGFR1 (7), and G protein-coupled receptor 54 (GPR54) $(8,9)$ underlie normosmic IHH (nIHH). Additionally, nasal embryonic LHRH factor (NELF) has been implicated in the pathogenesis of KS (10). Despite these advances, conundrums remain in understanding the genetic basis of IHH. For example, there is a puzzling clinical heterogeneity of the reproductive and

Nonstandard abbreviations used: D3, immunoglobulin-like domain 3; FGFR1, FGF receptor 1; GnRH, gonadotropin-releasing hormone; GNRHR, gonadotropin-releasing hormone receptor; IHH, idiopathic hypogonadotropic hypogonadism; KAL1, Kallmann syndrome 1 sequence; KS, Kallmann syndrome; NELF, nasal embryonic LHRH factor; $\mathrm{nIHH}$, normosmic IHH; SPR, surface plasmon resonance; T, testosterone. Conflict of interest: The authors have declared that no conflict of interest exists. Citation for this article: J. Clin. Invest. 117:457-463 (2007). doi:10.1172/JCI29884. nonreproductive phenotypes both within and across IHH families carrying identical single gene mutations $(4,6,11-14)$. Therefore, a given genotype at a single locus cannot reliably predict the phenotypic manifestations in any given member of affected families. Additionally, some mutations in genes accounting for $\mathrm{IHH}$, especially FGFR1, apparently demonstrate incomplete penetrance (4). Finally, defects in the identified genetic loci account for only a small percentage $(<30 \%)$ of cases. Thus, it is likely that other major IHH loci remain to be discovered and/or that the remaining (>70\%) cases are caused by the interplay of several contributing genes.

We hypothesized that IHH often involves defects in more than 1 gene. Herein we report evidence of IHH caused by the interaction of 2-gene defects (FGFR 1 and NELF in pedigree 1 and GNRHR and FGFR1 in pedigree 2). In addition, in vitro biochemical characterization of FGFR1 and NELF mutants is provided.

\section{Results}

Pedigree 1. Pedigree 1 has several affected members (Figure 1). The proband (no. 1-03) was referred to an endocrinologist at age 21 for failure to undergo puberty. His presentation was consistent with a severe KS phenotype. He was unvirilized, had eunuchoidal proportions (height, $176 \mathrm{~cm}$; span, $185 \mathrm{~cm}$ ), bilateral gynecomastia, microphallus, prepubertal testes $(2 \mathrm{ml}$; normal, $>12 \mathrm{ml}$ ), a repaired cleft lip/palate, and clinodactyly. His serum gonadotropin levels were undetectable, testosterone $(\mathrm{T})$ was $1 \mathrm{nmol} / \mathrm{l}$, and inhibin B $74 \mathrm{pg} / \mathrm{ml}$; otherwise, he had normal pituitary function and brain imaging. 


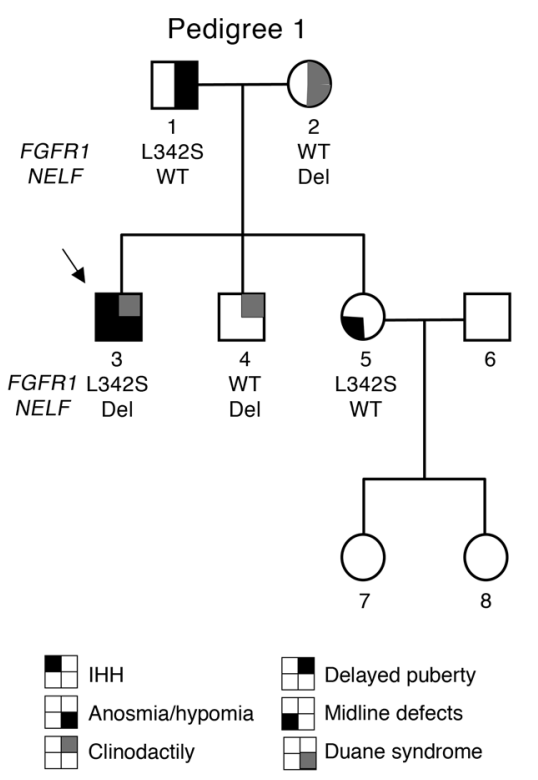

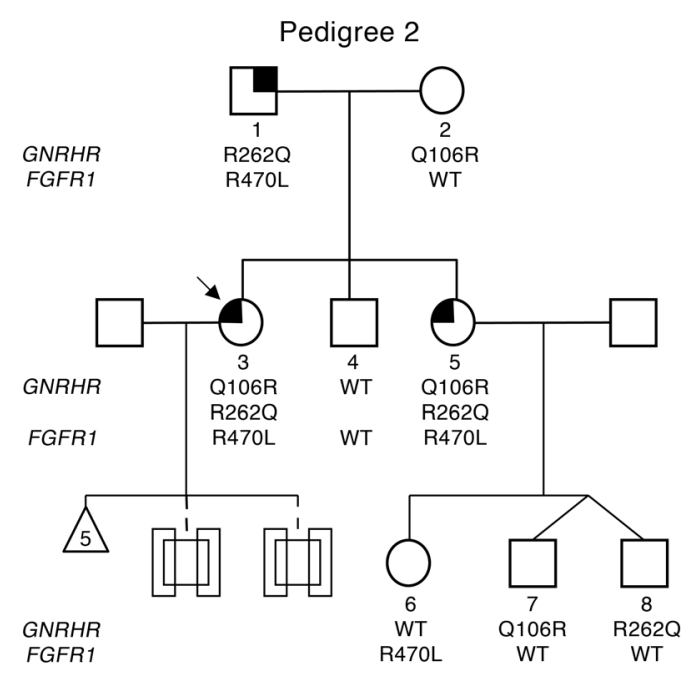

\section{Figure 1}

Identification of FGFR1 (p.L342S) and NELF (8-bp intronic deletion) mutations in pedigree 1; identification of GNRHR [p.Q106R] and [p.R262Q] and FGFR1 (p.R470L) mutations in pedigree 2. Only subjects harboring 2 gene defects have $\mathrm{IHH}$. Probands are identified by arrows; circles denote females, squares denote males. Del, $N E L F$ intronic deletion.
Formal testing revealed hyposmia (score of 29/40, below fifth percentile for his age) (15). Two subsequent years of gonadotropin therapy induced full virilization and sperm production. His father (no. 1-01) had a history of delayed puberty (growth spurt and full virilization after age 17) and congenital anosmia (score of 13/40), and his adult serum T was $18.1 \mathrm{nmol} / \mathrm{l}$. The proband's mother (no. 1-02) had clinodactyly and Duane ocular retraction syndrome and was menopausal. The proband's sister (no. 1-05) exhibited midline defects, including a bifid nose and high arched palate; the brother (no. 1-04) exhibited clinodactyly only. The mother and both siblings had normal puberty and a normal sense of smell as determined by formal testing.

Mutational analysis of the FGFR1 gene. The proband (no. 1-03) carries a unique heterozygous mutation $(\mathrm{c} .1025 \mathrm{~T} \rightarrow \mathrm{C}$ ) in exon 7 predicted to substitute a leucine for serine at position 342 (p.L342S) in the immunoglobulin-like domain 3 (D3) of FGFR1 (Figure 1, Figure 2, A and B, and Figure 3G). This change was also found in the affected father (no. 1-01) and the affected sister (no. 1-05) (Figure 1) but not in 200 white controls.

Structural and biochemical analysis of the L342S mutation implicate a loss of FGF8b signaling through FGFR1c in the pathology of $K S / I H H$. The isoforms FGFR1b and FGFR1c are generated by alternative splicing of exons $8 \mathrm{~A}$ and $8 \mathrm{~B}$, respectively (16). To date, FGFR1 mutations causing IHH have only been identified in exon 8B (17), implicating FGFR1c in the pathogenesis of IHH. Because L342 is highly conserved among the " $\mathrm{C}$ " splice isoforms of FGFR1-3 across species (Figure 2B), we used the FGFR2c-FGF8b crystallography model (18) to study L342S. The corresponding amino acid in FGFR2c, L343, is a key constituent in the hydrophobic

\section{Figure 2}

Schematic showing location of the 2 FGFR1 mutations and conservation of $L 342$ and $R 470$ residues across species and FGFRs. (A) The FGFR1 gene is located on chromosome 8p. FGFR1 contains 18 exons with intervening introns not drawn due to scale. SP, signal peptide; TM, transmembrane domain; TK, tyrosine kinase domain. (B) Comparison of L342 and R470 across species and within the FGFR family. groove of D3 and is extensively engaged by FGF8b (18) (Figure 3G). This leucine accounts for the unique binding specificity of FGF8b for the " $c$ " isoforms of FGFR1-3. Consistent with these structural data, surface plasmon resonance (SPR) analysis revealed a dramatic loss (20-fold) in the affinity of the L342S mutant for FGF8b (Figure $3, \mathrm{C}$ and $\mathrm{F}$ ), with only a small decrease (2-fold) in affinity for FGF1 (Figure 3, A and D) and (3-fold) FGF2 (Figure 3, B and E).

L342S FGFR1c missense mutation is a loss-of-function mutation. L6 myoblasts transiently expressing WT FGFR1c were treated with FGF8b, which induced a 6-fold increase in LUC reporter gene expression (Figure $3 \mathrm{H}$ ). In agreement with SPR results, the L342S FGFR1c was silent when expressed alone. As the KS subject harbored a heterozygous L342S mutation, we further coexpressed the WT FGFR1 and L342S in 1:1 and 1:2 ratios. The results are compatible with the hypothesis that this mutant acts as a dominant-negative mutation.

The variable degree of sexual maturation among family members carrying the same FGFR1 mutation led to further candidate

\section{A FGFR1}

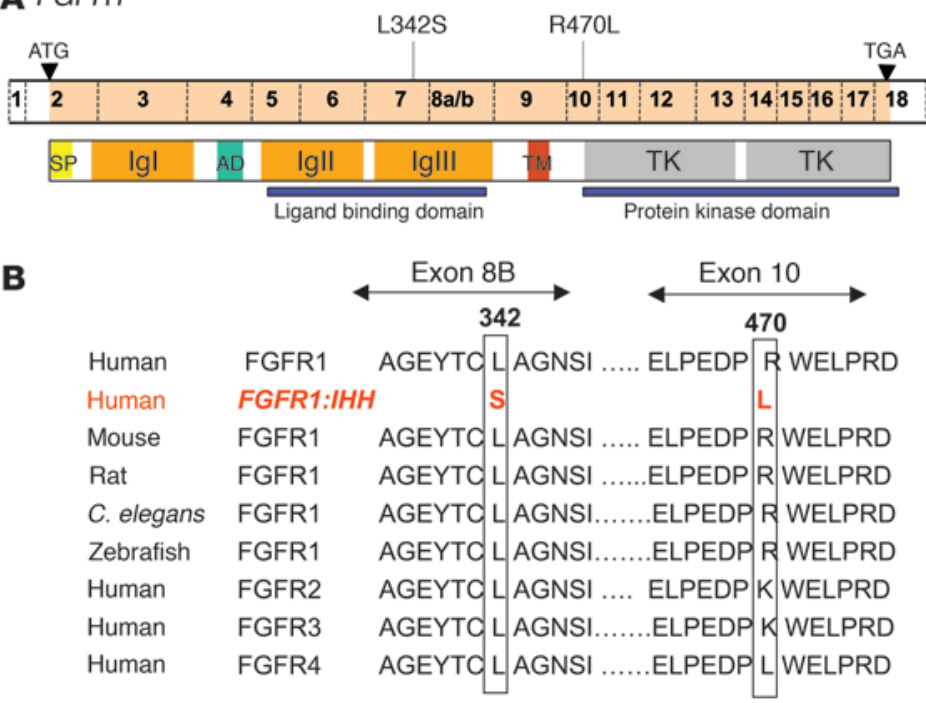



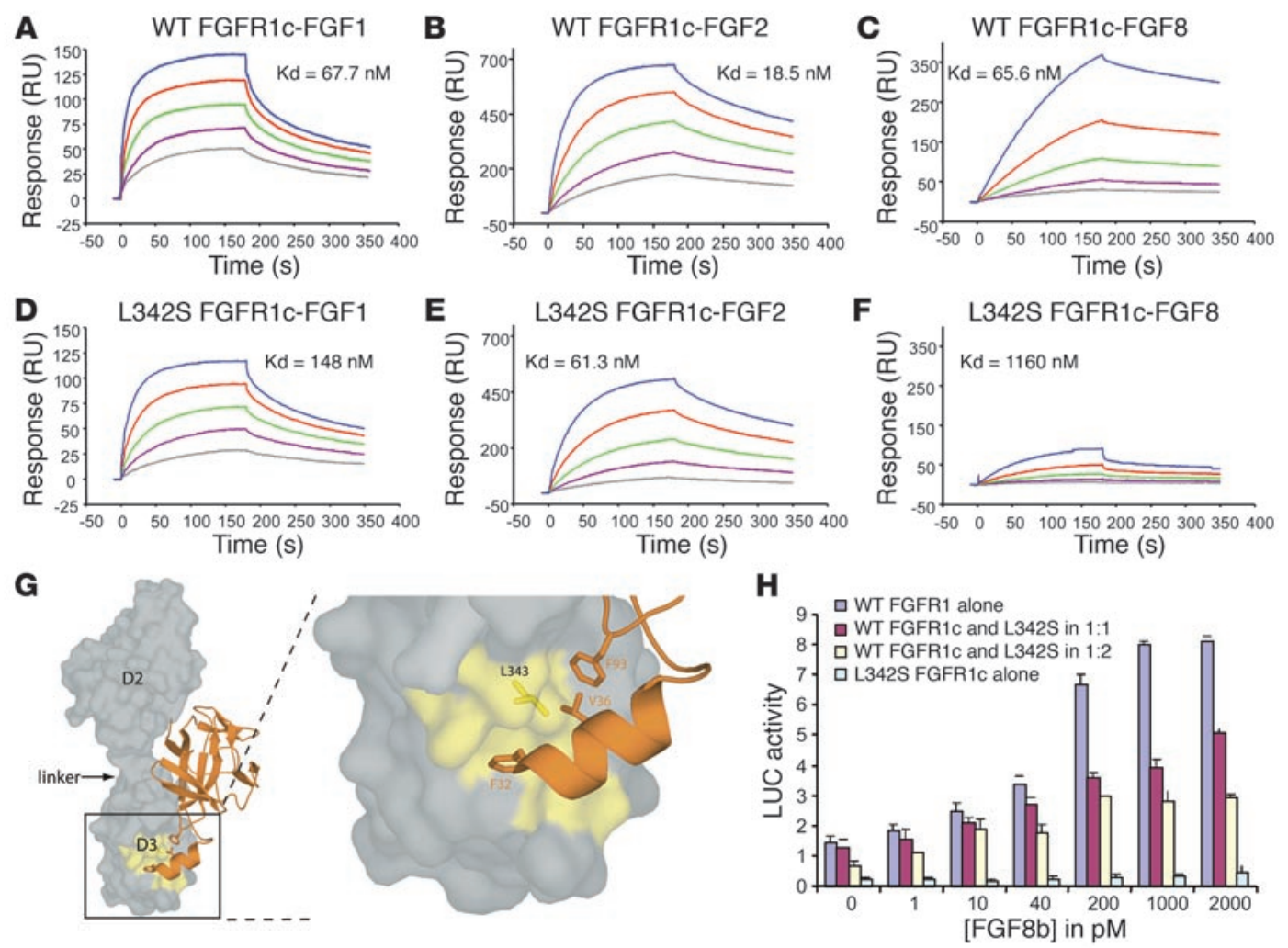

Figure 3

The L342S mutation dramatically reduces affinity of FGFR1c for FGF8b, implicating decreased FGF8b/FGFR1c signaling in the etiology of $\mathrm{KS} / \mathrm{IHH}$. (A-F) The L342S mutation reduces the affinity of FGFR1c for FGF8b. Varying concentrations of WT (A-C) or L342S FGFR1c mutant (D-F) were injected over a CM5 chip onto which FGF1 (A and D), FGF2 (B and E), and FGF8b (C and F) were immobilized. Analyte concentrations are indicated as follows: $31.25 \mathrm{nM}$ in gray, $62.5 \mathrm{nM}$ in violet, $125 \mathrm{nM}$ in green, $250 \mathrm{nM}$ in red, $500 \mathrm{nM}$ in blue. (G) The location of L343 in FGFR2c, the residue corresponding to L342 in FGFR1c, is mapped onto the FGF8b-FGFR2c structure (18). The L342S mutation should weaken key hydrophobic contacts between F32, V36, and F93 of FGF8b and D3 of FGFR. Gray: molecular surface of FGFR; orange: FGF8b. The side chains of selected residues are shown. The molecular surface of the hydrophobic groove of FGFR D3 (yellow) is rendered transparent so that the side chain of L343 (the residue corresponding to L342 of FGFR1c) is visible. (H) L342S FGFR1 heterozygous mutation is a loss-of-function mutation. WT and L342S FGFR1c were transiently transfected into L6 myoblasts with an FGFR1-responsive osteocalcin promoter luciferase construct. FGF8b treatment of WT FGFR1c induced a 6-fold increase in LUC reporter gene expression, while the L342S FGFR1c alone remained silent. The coexpression of the WT and L342S FGFR1c suggests that this mutation acts as a dominant negative.

gene screening and the identification of an additional deletion in $N E L F$ in the severely affected proband.

Mutational analysis of the NELF gene. A heterozygous 8-bp deletion ending 14 bp before exon 10 (c.1159-14_-22del) was identified in the proband (no. 1-03), his mother (no. 1-02), and his brother (no. 1-04) (Figure 4A). This deletion was not observed in 384 white controls.

RT-PCR. The HEK-293 cells transfected with the WT plasmid expressed NELF exons 8-11 at the expected size (291 bp). However, cells transfected with the 8-bp intronic deletion plasmid expressed an additional 257-bp splice form of NELF mRNA, lacking exon 10 (Figure 4C). The missplicing of mRNA lacking exon 10 results in a premature stop codon that predicts a truncated NELF protein product (p.Y376X) rather than the full-length product, which comprises 528 residues.

Immunobistology of FNC-C4 cells. Colocalization of both NELF and GnRH1 in FNC-B4 cells was demonstrated by immunohistochemistry (Figure 4D) and RT-PCR (data not shown).

Genotype-phenotype correlations. Proband no. 1-03 exhibited a severe KS phenotype with absent puberty, microphallus, hyposmia, undetectable serum luteinizing hormone and follicle stimulating hormone levels, hypogonadal T levels, and low inhibin B levels. He also had clinodactyly and cleft lip and palate. He harbored both a paternally derived heterozygous FGFR1 mutation (c.1025 T $\rightarrow$ C, p.L342S), resulting in weaker binding to FGF8b, and a maternally derived heterozygous 8-bp intronic deletion of NELF, resulting in a splicing defect of exon 10 and premature stop codon (Figures 1-4). While a single gene defect was associated with an attenuated phenotype (i.e., delayed puberty and anosmia in the father carrying L342S only), 2 mutant genes/gene products (FGFR1 and NELF) synergized to produce a more severe KS phenotype.

Pedigree 2. All members of the second, previously reported (14) pedigree were normosmic by formal smell testing, including 2 sisters affected with nIHH (Figure 1). The proband (no. 2-03) presented at 17 with primary amenorrhea, no breast development, short fourth metacarpals, and osteoporosis. She had undetectable serum gonadotropin and estradiol (E2) levels but otherwise normal pituitary function and cranial imaging. As previously reported, pulsatile GnRH induced ovulation in the proband, but she had 5 consecutive miscarriages (14). Her sister also presented at 18 with primary amenorrhea, absent breast development, and scoliosis. She had 2 success- 


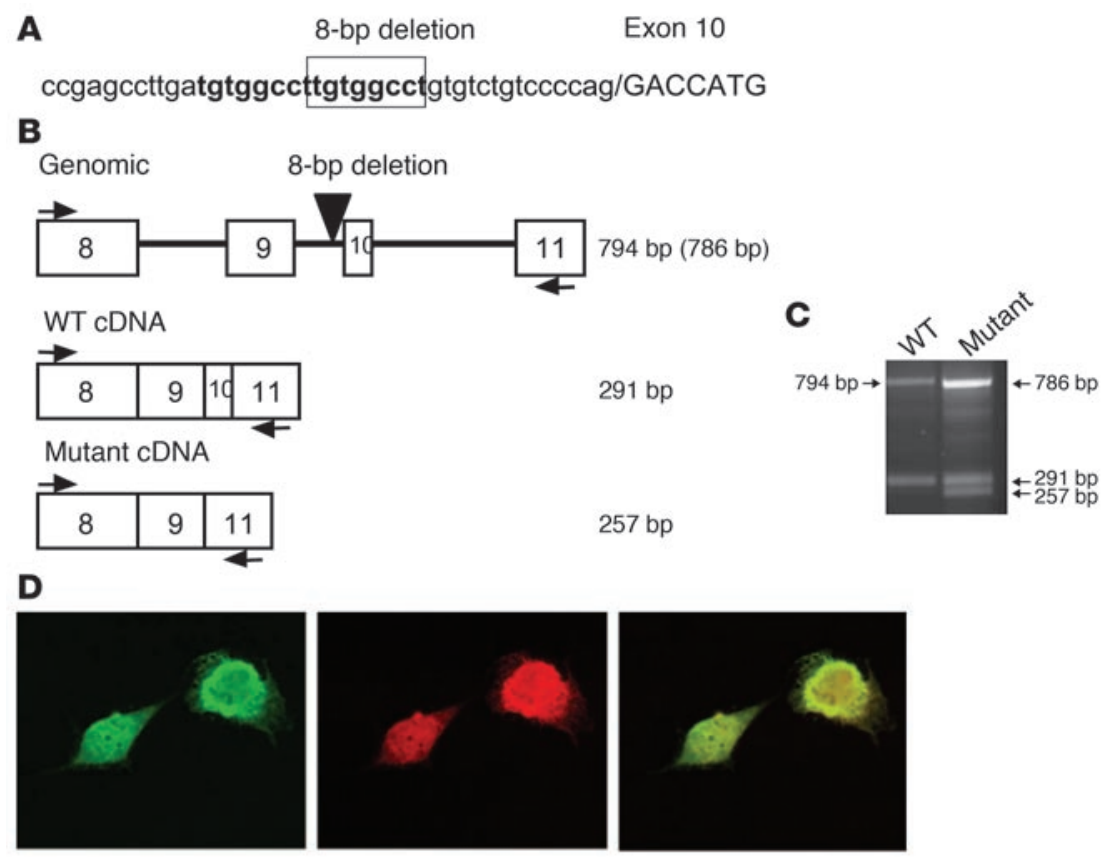

Figure 4

A 8-bp intronic NELF deletion results in missplicing of exon 10. (A) Direct DNA sequencing of cloned PCR products of the proband's genomic DNA revealed a heterozygous 8-bp deletion in intron 9. This deletion is part of a direct 8-bp repeat (tgtggcct) and occurs 14 bp upstream of the exon 10 acceptor $\left(5^{\prime}\right)$ splice site. The lower case part of the sequence indicates introns while the upper case indicates exons. (B) Predicted cDNAs in the exon 8-11 region of the $N E L F$ gene. The proband was found to have an 8-bp deletion in intron 9 (black triangle), 14 bp upstream of exon 10. (C) The result of RT-PCR using HEK-293 cell mRNA from cells transfected with WT NELF genomic construct containing exons 8-11 and the mutant NELF construct (8-bp deletion in intron 9). The HEK-293 cells transfected with the WT NELF construct show a normally spliced NELF exon 8-11 RT-PCR product, corresponding to the predicted size of $291 \mathrm{bp}$. The cells transfected with the mutant NELF construct, however, show an additional band of $257 \mathrm{bp}$ corresponding to the expected size of a transcript lacking exon 10. The RNA from cells transfected with either WT or mutant genomic construct show an additional product of $794 \mathrm{bp}$ (786 bp for the mutant construct), reflecting PCR amplification of the residual plasmid DNA. (D) Colocalization of NELF and GnRH1 in the olfactory epithelial cell line FNC-B4 by immunohistochemistry. Original magnification, $\times 150$.

ful pregnancies on gonadotropin therapy. Her daughter (no. 2-06) underwent normal puberty and has scoliosis, and male twins (nos. 2-07 and 2-08) were born without cryptorchidism or microphallus and have yet to undergo puberty (10 years old). The proband's father has a history of delayed puberty, scoliosis, and bilateral hearing loss. The brother was born with a cardiac septal defect and 3 fused cervical bones and went through normal puberty. The mother is menopausal but had normal reproductive function.

GNRHR mutational analysis. A compound heterozygous mutation in GNRHR [Q106R] and [R262Q] was identified in the proband (no. 2-03) and her sister (no. 2-05) (14). Both exhibited severe nIHH with absent puberty. Their father (no. 2-01) with a history of delayed puberty was heterozygous for R262Q, while the mother with no reproductive phenotype (no. 2-02) was heterozygous for Q106R. In addition, the brother (no. 2-04) and the niece (no. 2-06) were WT, while the twins (nos. 2-07 and 2-08) were heterozygous for Q106R and R262Q, respectively (Figure 1). In 1997, de Roux et al. described 2 siblings with partial nIHH as evidence by spontaneous testicular growth and active spermatogenesis in the male and
Tanner IV breast development in the female carrying the same compound heterozygous [Q106R] and [R262Q] mutation in the GNRHR gene. In vitro studies demonstrated that each mutant, [Q106R] and [R262Q], resulted in loss of function (6). The variable expressivity ranging from absent puberty to a partial puberty across families carrying the same compound heterozygous GNRHR mutation led to further candidate gene screening, which identified an additional FGFR1 mutation in our pedigree.

FGFR1 mutational analysis. We identified a heterozygous mutation in FGFR1 (c.1409 $\mathrm{G} \rightarrow \mathrm{T}$ ) in exon 10 in both nIHH sisters. This nucleotide change is predicted to substitute an arginine for leucine at position 470 (p.R470L) (Figure 2A). Screening of the entire pedigree revealed this change only in the father with delayed puberty (no. 2-01) and the unaffected niece (no. 2-06) (Figure 1). R470 is conserved across species (Figure 2B), and R470L was not detected in 200 white controls.

The R470L mutation reduces the tyrosine kinase activity of FGFR1. In the FGFR1 kinase structure, arginine 470 (R470) lies at the junction between the kinase domain and the juxtamembrane region (Figure $2 \mathrm{~A}$ ). The side chain of R470 makes 3 hydrogen bonds with D468; the latter engages in a hydrogen bond with K536 from the $\alpha \mathrm{C}$ helix (Figure 5A) (19). Crystal structures of unphosphorylated and phosphorylated kinase domains show distinct and reversible movements of the $\alpha \mathrm{C}$ helix during the kinase activation/inactivation cycle. R470 both facilitates the conformation of the juxtamembrane/kinase region and contributes to proper $\alpha \mathrm{C}$ positioning. Therefore, the R470L mutation should negatively impact the tyrosine kinase activity of FGFR1. Indeed, comparison of WT FGFR1 and the R470L mutant revealed a marked decrease in the tyrosine kinase activity in the mutant, indicating a loss of function (Figure 5B).

Genotype-phenotype correlations. The sisters severely affected with $\mathrm{nIHH}$ harbor both a compound heterozygous mutation in the GNRHR gene [Q106R] and [R262Q] and a heterozygous FGFR1 mutation (R470L) - therefore a triallelic pattern of inheritance. The additional FGFR1 mutation could explain the variable phenotypic expressivity seen between pedigrees harboring the same compound heterozygous GNRHR mutation (6). Interestingly, the father (no. 2-01), with a combination of 2 heterozygous mutant alleles (GNRHR and FGFR1), had delayed puberty, while other family members carrying only 1 mutant allele display a normal reproductive phenotype.

\section{Discussion}

IHH appears to follow the pattern of several disorders that were initially thought to be monogenic but have subsequently proven to be caused by or modulated by more than 1 gene defect $(20,21)$. In contrast to polygenic traits, these oligogenic disorders involve the synergistic action of mutant alleles at a small number of loci. This report expands our understanding of the genetics of IHH and 


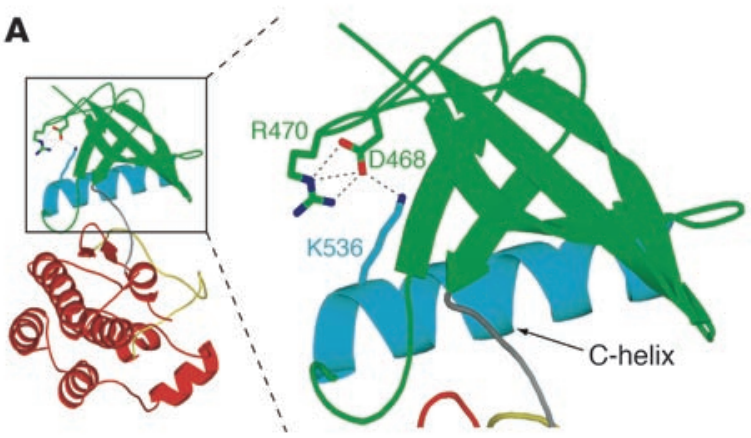

B

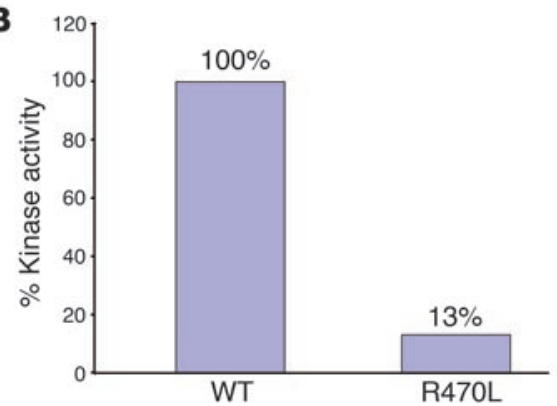

Figure 5

The R470L FGFR1 mutation is loss-of-function. (A) The $R \rightarrow L$ substitution abolishes hydrogen bonds that play a role in the positioning of the C-helix of FGFR1. The unphosphorylated "low-activity" form of the FGFR1 kinase domain (PDB ID: 1FGK) is represented as a ribbon diagram. The N-lobe of the FGFR1 kinase is shown in green, with the exception of the $\alpha \mathrm{C}$ helix, which is in blue. Red: C-lobe of FGFR1 kinase; gray: linker connecting the $\mathrm{N}$ - and $\mathrm{C}$-lobes; yellow: activation loop in the C-lobe. Selected residues are shown, and hydrogen bonds are represented as dashed lines. R470 indirectly contributes to the proper positioning of $\alpha \mathrm{C}$ in the kinase domain and hence kinase regulation by engaging in 3 hydrogen bonds with $\mathrm{D} 468$, which in turn engages in a hydrogen bond with K536 from $\alpha$ C helix. (B) The R470L mutation reduces the tyrosine kinase activity of FGFR1.

suggests oligogenicity may underly other monogenic disorders also characterized by incomplete penetrance and variable phenotypes within and between families.

IHH is currently described as a monogenic disorder resulting in defective GnRH secretion or action, with 7 loci implicated in the pathogenesis of the disease to date. However, the genotype-phenotype correlations from specific mutations in different loci have been imprecise. An illustrative example is FGFR1. Numerous heterozygous FGFR1 mutations underlie cases of KS (4, 13, 22-25) and $\mathrm{nIHH}(7$, 26), characterized by marked phenotypic variability both within and between families and apparent incomplete penetrance. Furthermore, although FGFR1 mutations are known as causing an autosomal dominant form of $\mathrm{IHH}$, there is a report of $1 \mathrm{KS}$ subject with a very severe phenotype harboring a homozygous FGFR 1 mutation (4). These data suggest a dosage effect of the FGFR1 mutant alleles and the existence of modifier genes and/or environmental effects leading to phenotypic variability across and within families.

Herein, we report a proband who carries heterozygous mutations in both FGFR1 and NELF and exhibits a severe KS phenotype with cleft palate (pedigree 1). The FGFR1 mutation, L342S, alters the spectrum of FGF binding by decreasing FGF8b binding specifically. These results were corroborated by our reporter gene assay, which showed the L342S mutation to be a loss-of-function mutation, potentially having a dominant-negative effect in the heterozygous state. In addition, these data implicate FGF8b as a key ligand for FGFR1c in the pathogenesis of KS. These findings are in accordance with studies on mice with a hypomorphic Fgf8 allele, which exhibited defected nasal cavity development and olfactory bulbs dysgenesis, a phenotype similar to that observed in the Fgfr1 conditional knockout mouse (27-29). Therefore, we believe that the L342S FGFR1 mutation contributed to both the $\mathrm{KS}$ and the craniofacial phenotype of the proband. However, the KS phenotype failed to segregate with the L342S mutation, as only the proband exhibited KS. Interestingly, he carried an additional heterozygous intronic NELF deletion resulting in missplicing of exon 10 and a premature stop codon. NELF plays a key role in GnRH and olfactory neuron outgrowth (30-32) and is colocalized with GnRH1 in human olfactory stem cells (Figure 4D). NELF has previously been implicated as a potential locus for IHH in an affected hypogonadotropic patient with a unique heterozygous mutation (c.1438A $\rightarrow$ G; p.T480A), though the biology of this mutant remains unexplored (10). Interestingly, the mother (no. 1-02), who carried the NELF mutation only, had Duane ocular retraction syndrome (OMIM 126800), a disorder of neuronal migration that has been associated with KS (33). Therefore, we believe that the heterozygous mutations in both the NELF and the FGFR1 gene synergized to cause severe KS in the proband, while individuals carrying only 1 of the 2 mutations do not present with KS.

Since 1997, GNRHR mutations have been known to cause a recessive form of nIHH. However, unexplained variable expressivity of IHH across pedigrees carrying the same compound heterozygous mutation in GNRHR $(6,14)$ led us to screen further candidate genes. As a result, we identified a triallelic mode of inheritance for IHH in pedigree 2 . Subjects carrying only 1 mutant allele (FGFR1 or GNRHR) had a normal reproductive phenotype. Further, the individual (no. 1-01) carrying both heterozygous FGFR1 and GNRHR mutations had only a mild reproductive phenotype (delayed puberty), while the 2 sisters harboring both a loss-of-function compound heterozygous mutation in GNRHR and a loss-offunction heterozygous FGFR1 mutation (R470L) had severe IHH phenotypes. Although IHH was initially thought to be transmitted as a recessive trait, this pedigree indicates a triallelic digenic inheritance of IHH, suggesting that in some families, more than 2 mutant alleles might be required to manifest IHH. Similar patterns of mutation lead to Bardet-Biedl syndrome (34).

Only $30 \%$ of IHH subjects studied to date harbor a mutation in 1 of the genes known to cause $\mathrm{IHH}$, and thus it is not possible to infer the frequency of oligogenicity in IHH. The concept of digenicity was evoked but not proven in a recent report that described a KS subject carrying both a KAL1 missense mutation and a PROKR2 missense mutation (5). However, the functionality of these mutants was not explored, and no data were presented on the pedigree of this subject.

The discovery of oligogenic traits in IHH raises both conceptual and practical issues. Because $\mathrm{IHH}$ is no longer considered to be a monogenic disorder, the transmission of a trait through families is no longer synonymous with the transmission of 1 specific mutant allele. Therefore, we must rethink our current approach in studying the genetics of $\mathrm{IHH}$, separating the segregation of a trait from the segregation of a specific mutant allele (21). The results of this study indicate that labeling mutant alleles as dominant or recessive is often an oversimplification. Oligogenicity also has implications for genetic counseling regarding IHH. Finally, the question remains 
as to whether the variability of expressivity in IHH is controlled by a small number of major loci or a large number of minor loci.

The molecular basis of oligogenicity is poorly understood. To date, one of the best examples of the molecular basis of digenic inheritance is the direct interaction of the mutants retinal outer segment membrane protein 1 and retinal degeneration slow, which causes retinitis pigmentosa (35). The 2 mutants combine to prevent the formation of a tetrameric complex that is important for the integrity of the photoreceptors. Further examples in which synergistic mutations at different loci cause a disease are found in diseases characterized by mutations in receptor-ligand pairs, such as Hirschsprung disease (RET and GDNF) (36). In addition, indirect interactions between 2 mutated proteins have been reported in severe insulin resistance where PPARG and PPP1R3A mutants are expressed in 2 different tissues (37).

In contrast, little is known about the molecular pathways whereby the 2 pairs of mutant proteins (NELF and FGFR1; GnRHR and FGFR1) contribute to the IHH phenotype. FGFR1 and NELF are both expressed in GnRH neurons $(30,38,39)$. Therefore, the 2 mutant proteins may act at different levels of the same intracellular pathway and may quantitatively contribute to its progressive dysfunction until a critical threshold is reached, thus producing the disease phenotype. Alternatively, these 2 proteins may participate in a multiprotein complex that becomes progressively compromised by the additional mutations. In the other gene pair (FGFR1 and GNRHR), the FGFR1 mutant is anticipated to reduce the number of $\mathrm{GnRH}$ neurons, as evidenced by a mouse model with targeted dominant-negative FGFR1 in the GnRH neurons (40). These mice, although fertile, display a 30\% decrease in the number of GnRH neurons in the hypothalamus. Conversely, the GNRHR mutants result in GnRH resistance that would require a compensatory increase in $\mathrm{GnRH}$ to restore reproductive function. Such a putative compensatory mechanism would be impaired in the individuals with an additional FGFR1 mutation. Thus, deficient GnRH migration, secretion, and/or action could be caused by the interaction of these mutants in the same or different developmental pathways, leading to subtle amplification effects of each mutant protein.

This report expands our understanding of the genetics of this disorder by demonstrating that IHH can be caused by the combination of gene defects. The complexity of this genetic model implies a revision of the genetic terminology of IHH. Furthermore, these data predict that mutational analysis in multiple genes for seemingly monogenic disorders will become increasingly frequent and may lead to greater accuracy in phenotypic predictions. In the case of $\mathrm{IHH}$, studying the interaction of a small number of key loci will undoubtedly be key to understanding some of the broader variability of the timing of normal puberty.

\section{Methods}

Mutational analysis. Genetic studies were approved by Partners Healthcare Human Research Committee, and all participants provided written informed consent.

Whole blood samples were obtained from subjects and genomic DNA extracted. Exons and exon-intron boundaries were amplified using standard PCR techniques for GNRHR (Genbank accession number NM_001012763 [ref. 41]); KAL1 (accession number NM_000216 [ref. 42]); GPR54 (accession number NM_032551 [ref. 9]); FGFR1 (accession number BC018128 [ref. 4]); and NELF (accession number ENSG00000165802 [ref. 10]). Nonsense changes resulting in a truncated protein, frameshift, insertion, or deletion were categorized as definite mutations. Nucleotide changes, which were (a) absent from the Single Nucleotide Polymorphism database (http://www.ncbi.nlm.nih.gov/projects/SNP/) and expressed sequence tags and (b) absent in at least 170 ethnically matched healthy controls were identified as mutations. All genes and proteins are described using standard nomenclature (43).

Control population. To determine whether the observed bp changes in the genes cited above were normal variants, a cohort of adult healthy white subjects $(n \geq 200)$ were screened.

Structural analysis of the effects of FGFR 1 mutations on FGFR1 function. Crystal structures of the FGFR1 kinase domain (Research Collaboratory for Structural Bioinformatics Protein Data Bank identification [PDB ID]: 1FGK) (19) and the extracellular ligand binding region of FGFR2c in complex with FGF8b and heparin oligosaccharide (PDB ID: 2FDB) (18) were used to map the potential effects of the mutations. Crystal structures were visualized using program $\mathrm{O}$ (44).

SPR analysis. Real-time biomolecular interactions between WT and L342S mutant FGFR1c extracellular domains with FGF1, FGF2, and FGF8 were characterized with a Biacore 3000 instrument as previously described (18).

Plasmids and in vitro mutagenesis. Human WT FGFR1c cDNA (NM_000604) was subcloned into a pcDNA3.1 $1^{+}$expression vector (Invitrogen). The FGFR1c L342S mutation was introduced into the human FGFR1c cDNA by site-directed mutagenesis (QuikChange Site-Directed Mutagenesis Kit; Stratagene) and subcloned into pcDNA3. $1^{+}$using HindIII and XhoI restriction sites.

Reporter gene assay. To demonstrate that the heterozygous L342S mutation was a loss-of-function mutation, we used an FGF reporter bioassay. WT FGFR1c and L342S FGFR1c were transfected along with an osteocalcin FGF response element (OCFRE) promoter/luciferase reporter (45) into L6 myoblasts, a cell line largely devoid of endogenous FGFRs and FGFs (46, 47). L6 myoblasts were maintained in DMEM containing penicillin (100 U/l), streptomycin $(100 \mu \mathrm{g} / \mathrm{l})$, and $10 \% \mathrm{FCS}$ (Invitrogen). A total of $4 \times 10^{4}$ cells per well in 24-well plates were seeded and 24 hours later transiently transfected with a total of $400 \mathrm{ng}$ of DNA consisting of $100 \mathrm{ng}$ of WT FGFR1c cDNA, $30 \mathrm{ng}$ of OCFRE reporter gene, and 0, 100, and $200 \mathrm{ng}$ of the mutated FGFR1c (L342S) and empty vector using FuGENE 6 Reagent (Roche Diagnostics). Twenty-four hours after transfection, the cells were treated with increasing doses of FGF8b (from 0 to 2,000 pM) in culture medium containing $0.1 \% \mathrm{FCS}$ and $1 \mu \mathrm{g} / \mathrm{ml}$ heparin. Following 16 hours of incubation, the cells were lysed, and luciferase activities were measured using Promega Luciferase Assay System (Promega). Since Renilla and $\beta$-galactosidase were induced by FGFs, the luciferase data are reported directly. Experiments were performed in triplicate and repeated once. The data are reported as mean $\pm \mathrm{SD}$ of a representative experiment performed in triplicate.

Kinase assay. The tyrosine autophosphorylation activity of the WT and R470L mutant FGFR1 kinase domains were quantified using a continuous spectrophotometric assay as previously described (48).

Generation of the NELF mutant construct. To assess the effect of the intronic deletion of the NELF gene on exon splicing, WT and mutant genomic NELF constructs were made in the expression vector PCR3.1 and transfected into HEK-293 cells (Lipofectamine 2000; Invitrogen). Oligonucleotide primers (F5': CAGTGACCTGCAGAGCTC-3' and R5': CCAGATCTTGGCTCCCTTGTG-3') spanning the genomic sequence from within exons 8 and 11 were used to amplify, via PCR, a fragment of WT DNA (794 bp) and mutant DNA (786 bp in the deleted allele) (Figure 4B). RT-PCR was expected to yield a 291-bp product from a $N E L F \mathrm{cDNA}$ with correct splicing of exons 9 and 10.

Immunobistochemistry of FNC-B4 cells. FNC-B4 cells, isolated from human fetal olfactory epithelia, have characteristics of both secretory and migratory GnRH neurons $(39,49)$. FNC-B4 cells were stained with either GnRH1 or NELF antibodies $(30,49)$. Secondary antibodies conjugated with either Texas red or FITC were used to visualize GnRH1 and NELF staining. 


\section{Acknowledgments}

We thank David Ornitz for his kind gift of the OCFRE luciferase reporter. This work was funded by NIH grants DE13686, HD15788, and HD028138.

Received for publication July 27, 2006, and accepted in revised form November 27, 2006

1. Franco, B., et al. 1991. A gene deleted in Kallmann's syndrome shares homology with neural cell adhesion and axonal path-finding molecules. Nature. 353:529-536.

2. Legouis, R., et al. 1991. The candidate gene for the $\mathrm{X}$-linked Kallmann syndrome encodes a protein related to adhesion molecules. Cell. 67:423-435.

3. Hardelin, J.P., et al. 1993. Heterogeneity in the mutations responsible for $\mathrm{X}$ chromosome- linked Kallmann syndrome. Hum. Mol. Genet. 2:373-377.

4. Dode, C., et al. 2003. Loss-of-function mutations in FGFR1 cause autosomal dominant Kallmann syndrome. Nat. Genet. 33:463-465.

5. Dodé, C., et al. 2006. Kallmann syndrome: mutations in the genes encoding prokineticin- 2 and prokineticin receptor-2. PLoS Genet. 2:e175.

6. de Roux, N., et al. 1997. A family with hypogonadotropic hypogonadism and mutations in the gonadotropin-releasing hormone receptor. N. Engl. J. Med. 337:1597-1602.

7. Pitteloud, N., et al. 2006. Mutations in fibroblast growth factor receptor 1 cause both Kallmann syndrome and normosmic idiopathic hypogonadotropic hypogonadism. Proc. Natl. Acad. Sci. U. S. A. 103:6281-6286.

8. de Roux, N., et al. 2003. Hypogonadotropic hypogonadism due to loss of function of the KiSS1derived peptide receptor GPR54. Proc. Natl. Acad. Sci.U. S. A. 100:10972-10976.

9. Seminara, S.B., et al. 2003. The GPR54 gene as a regulator of puberty. N. Engl. J. Med. 349:1614-1627.

10. Miura, K., Acierno, J.S., Jr., and Seminara, S.B. 2004. Characterization of the human nasal embryonic LHRH factor gene, NELF, and a mutation screening among 65 patients with idiopathic hypogonadotropic hypogonadism (IHH). J. Hum. Genet. 49:265-268.

11. de Roux, N., et al. 1999. The same molecular defects of the gonadotropin-releasing hormone receptor determine a variable degree of hypogonadism in affected kindred. J. Clin. Endocrinol. Metab. 84:567-572.

12. Massin, N., et al. 2003. X chromosome-linked Kallmann syndrome: clinical heterogeneity in three siblings carrying an intragenic deletion of the KAL-1 gene. J. Clin. Endocrinol. Metab. 88:2003-2008.

13. Pitteloud, N., et al. 2005. Reversible kallmann syndrome, delayed puberty, and isolated anosmia occurring in a single family with a mutation in the fibroblast growth factor receptor 1 gene. J. Clin. Endocrinol. Metab. 90:1317-1322.

14. Seminara, S.B., et al. 2000. Successful use of pulsatile gonadotropin-releasing hormone $(\mathrm{GnRH})$ for ovulation induction and pregnancy in a patient with GnRH receptor mutations. J. Clin. Endocrinol. Metab. 85:556-562.

15. Mayston, M.J., Harrison, L.M., and Stephens, J.A. 1999. A neurophysiological study of mirror movements in adults and children. Ann. Neurol. 45:583-594.

16. Eswarakumar, V.P., Lax, I., and Schlessinger, J. 2005. Cellular signaling by fibroblast growth factor receptors. Cytokine Growth Factor Rev. 16:139-149.

17. Zitzmann, M., and Nieschlag, E. 2000. Hormone substitution in male hypogonadism. Mol. Cell.

Address correspondence to: Nelly Pitteloud, Reproductive Endocrine Unit, BHE 5, Massachusetts General Hospital, Boston, 02114 Massachusetts, USA. Phone: (617) 724-1830; Fax: (617) 726-5357; E-mail: npitteloud@partners.org.

Moosa Mohammadi and William Crowley Jr. contributed equally to this work.

Endocrinol. 161:73-88

18. Olsen, S.K., et al. 2006. Structural basis by which alternative splicing modulates the organizer activity of FGF8 in the brain. Genes Dev. 20:185-198.

19. Mohammadi, M., Schlessinger, J., and Hubbard, S.R. 1996. Structure of the FGF receptor tyrosine kinase domain reveals a novel autoinhibitory mechanism. Cell. 86:577-587.

20. Carlton, V.E., et al. 2003. Complex inheritance of familial hypercholanemia with associated mutations in TJP2 and BAAT. Nat. Genet. 34:91-96.

21. Badano, J.L., and Katsanis, N. 2002. Beyond Mendel: an evolving view of human genetic disease transmission. Nat. Rev. Genet. 3:779-789.

22. Albuisson, J., et al. 2005. Kallmann syndrome: 14 novel mutations in KAL1 and FGFR1 (KAL2). Hum Mutat. 25:98-99.

23. Sato, N., et al. 2004. Clinical assessment and mutation analysis of Kallmann syndrome 1 (KAL1) and fibroblast growth factor receptor 1 (FGFR1, or KAL2) in five families and 18 sporadic patients. J. Clin. Endocrinol. Metab. 89:1079-1088.

24. Zenaty, D., et al. 2006. Paediatric phenotype of Kallmann syndrome due to mutations of fibroblast growth factor receptor 1 (FGFR1). Mol. Cell. Endocrinol. 254-255:78-83.

25. Pitteloud, N., et al. 2006. Mutations in fibroblast growth factor receptor 1 cause Kallmann syndrome with a wide spectrum of reproductive phenotypes. Mol. Cell. Endocrinol. 254-255:60-69.

26. Trarbach, E.B., et al. 2006. Novel fibroblast growth factor receptor 1 mutations in patients with congenital hypogonadotropic hypogonadism with and without anosmia. J. Clin. Endocrinol. Metab. 91:4006-4012.

27. Hebert, J.M., Lin, M., Partanen, J., Rossant, J., and McConnell, S.K. 2003. FGF signaling through FGFR1 is required for olfactory bulb morphogenesis. Development. 130:1101-1111.

28. Meyers, E.N., Lewandoski, M., and Martin, G.R. 1998. An Fgf8 mutant allelic series generated by Cre- and Flp-mediated recombination. Nat. Genet. 18:136-141.

29. Kawauchi, S., et al. 2005. Fgf8 expression defines a morphogenetic center required for olfactory neurogenesis and nasal cavity development in the mouse. Development. 132:5211-5223.

30. Kramer, P.R., and Wray, S. 2000. Novel gene expressed in nasal region influences outgrowth of olfactory axons and migration of luteinizing hormone-releasing hormone (LHRH) neurons. Genes Dev. 14:1824-1834.

31. Wray, S. 2002. Molecular mechanisms for migration of placodally derived GnRH neurons. Chem. Senses. 27:569-572.

32. Gonzalez-Martinez, D., et al. 2004. Anosmin-1 modulates fibroblast growth factor receptor 1 signaling in human gonadotropin-releasing hormone olfactory neuroblasts through a heparan sulfate-dependent mechanism. J. Neurosci. 24:10384-10392.

33. Cordonnier, M., Hanozet, V., Van Nechel, C., Fery, F., and Aberkane, J. 1990. Bilateral Duane syndrome associated with hypogonadotropic hypogonadism and anosmia (Kallmann syndrome) [In French]. Bull. Soc. Belge Ophtalmol. 239:29-35.

34. Beales, P.L., et al. 2001. Genetic and mutational analyses of a large multiethnic Bardet-Biedl cohort reveal a minor involvement of BBS6 and delineate the critical intervals of other loci. Am. J. Hum. Genet. 68:606-616.

35. Kajiwara, K., Berson, E.L., and Dryja, T.P. 1994. Digenic retinitis pigmentosa due to mutations at the unlinked peripherin/RDS and ROM1 loci. Science. 264:1604-1608.

36. Parisi, M.A., and Kapur, R.P. 2000. Genetics of Hirschsprung disease. Curr. Opin. Pediatr. 12:610-617.

37. Savage, D.B., et al. 2002. Digenic inheritance of severe insulin resistance in a human pedigree. Nat. Genet. 31:379-384.

38. Gill, J.C., Moenter, S.M., and Tsai, P.S. 2004. Developmental regulation of gonadotropin-releasing hormone neurons by Fgf signaling. Endocrinology. 145:3830-3839.

39. Romanelli, R.G., et al. 2004. Expression and function of gonadotropin-releasing hormone ( $\mathrm{GnRH})$ receptor in human olfactory $\mathrm{GnRH}$-secreting neurons: an autocrine $\mathrm{GnRH}$ loop underlies neuronal migration. J. Biol. Chem. 279:117-126.

40. Tsai, P.S., et al. 2005. Targeted expression of a dominant-negative fibroblast growth factor (FGF) receptor in gonadotropin-releasing hormone $(\mathrm{GnRH})$ neurons reduces FGF responsiveness and the size of GnRH neuronal population. Mol. Endocrinol. 19:225-236.

41. Beranova, M., et al. 2001. Prevalence, phenotypic spectrum, and modes of inheritance of gonadotropin-releasing hormone receptor mutations in idiopathic hypogonadotropic hypogonadism. J. Clin. Endocrinol. Metab. 86:1580-1588.

42. Oliveira, L.M., et al. 2001. The importance of autosomal genes in Kallmann syndrome: genotype-phenotype correlations and neuroendocrine characteristics. J. Clin. Endocrinol. Metab. 86:1532-1538.

43. Antonarakis, S.E. 1998. Recommendations for a nomenclature system for human gene mutations. Nomenclature Working Group. Hum. Mutat. 11:1-3.

44. Mohammadi, M., et al. 1996. Identification of six novel autophosphorylation sites on fibroblast growth factor receptor 1 and elucidation of their importance in receptor activation and signal transduction. Mol. Cell. Biol. 16:977-989.

45. Newberry, E.P., Boudreaux, J.M., and Towler, D.A. 1996. The rat osteocalcin fibroblast growth factor (FGF)-responsive element: an okadaic acid-sensitive, FGF-selective transcriptional response motif. Mol. Endocrinol. 10:1029-1040.

46. Olwin, B.B., and Hauschka, S.D. 1989. Cell type and tissue distribution of the fibroblast growth factor receptor. J. Cell. Biochem. 39:443-454.

47. Roghani, M., et al. 1994. Heparin increases the affinity of basic fibroblast growth factor for its receptor but is not required for binding. J. Biol. Chem. 269:3976-3984.

48. Barker, S.C., et al. 1995. Characterization of pp60csrc tyrosine kinase activities using a continuous assay: autoactivation of the enzyme is an intermolecular autophosphorylation process. Biochemistry. 34:14843-14851.

49. Vannelli, G.B., et al. 1995. Neuroblast long-term cell cultures from human fetal olfactory epithelium respond to odors. J. Neurosci. 15:4382-4394. 